Präv Gesundheitsf 2020 · 15:399-404 https://doi.org/10.1007/s11553-020-00761-5 Eingegangen: 26. November 2019

Angenommen: 8. Januar 2020

Online publiziert: 31. Januar 2020

(c) Der/die Autor(en) 2020

Felix Zastrow' ${ }^{1}$ Alvia Killenberg' ${ }^{1}$ Cornelia Klünder' Nadine Ladebeck $^{2}$. Sandra Langner ${ }^{1} \cdot$ Katrin Neher $^{1} \cdot$ Maria Schimmelpfennig ${ }^{2} \cdot$ Tina Zeiler $^{2}$. Kerstin Baumgarten ${ }^{2} \cdot$ Holger Hassel $^{1}$

${ }^{\prime}$ Institut für angewandte Gesundheitswissenschaften (laG), Hochschule für angewandte Wissenschaften Coburg, Coburg, Deutschland

${ }^{2}$ Fachbereich Soziale Arbeit, Gesundheit und Medien, Hochschule Magdeburg-Stendal, Magdeburg, Deutschland

\title{
Intervention zur Förderung der Gesundheitskompetenz älterer Menschen - Ergebnisse des Projekts „GeWinn"
}

\section{Hinführung}

Die Gesundheitskompetenz (GK) bzw. Health Literacy (HL) ist bei einer Vielzahl älterer Menschen ab 65 Jahren in Deutschland eingeschränkt [7-10, 12]. Das Projekt GeWinn strebte an, die GK von Menschen ab 60 Jahren mit Hilfe von selbstständig arbeitenden Gruppen wirksam zu fördern. Daraus entwickelte sich die Frage, ob mit Hilfe eines 53-wöchigen Gruppenprogramms mit Peer-Moderation die GK älterer Menschen verbessert werden kann.

\section{Hintergrund}

Die GK umfasst „das Wissen, die Motivation und die Kompetenzen von Menschen, relevante Gesundheitsinformationen in unterschiedlicher Form $\mathrm{zu}$ finden, $\mathrm{zu}$ verstehen, zu beurteilen und anzuwenden, um im Alltag in den Domänen der Krankheitsbewältigung, der Krankheitsprävention und der Gesundheitsförderung Urteile fällen und Entscheidungen treffen zu können, die ihre Lebensqualität während des gesamten Lebensverlaufs erhalten oder verbessern" [10]. In Deutschland weisen nur $3 \%$ der über 65-Jährigen ein exzellentes GK-Niveau auf. Etwas mehr als die Hälfte $(51,1 \%)$ der älteren Menschen verfügt über ein problematisches, $15,2 \%$ sogar über ein unzureichendes
GK-Niveau. Dieser Bevölkerungsgruppe fällt insbesondere das Finden sowie das Beurteilen von Gesundheitsinformationen schwer [7-10, 12]. Gleichzeitig leiden 60,2\% der Frauen und 53,8\% der Männer über 65 Jahren an (mindestens) einer chronischen Erkrankung [6]. Eine eingeschränkte GK geht meist mit einem schlechten subjektiven Gesundheitszustand, geringeren Ressourcen für das Selbstmanagement von chronischer Krankheit und einem geringen subjektiven Wohlbefinden einher [1, 3, 8, 11]. Evidenzbasierte Interventionen, insbesondere für vulnerable Zielgruppen, fehlen weitgehend [7, 9].

Vor diesem Hintergrund wurde das vom Bundesministerium für Bildung und Forschung geförderte Projekt „GeWinn - Gesund älter werden mit Wirkung. Health Literacy für mehr Lebensqualität und soziale Integration“ generiert. Durch eine partizipative Entwicklung und Durchführung einer Intervention mit Peer-Moderation sollte die GK von Frauen und Männern ab 60 Jahren in den Bereichen Gesundheitsförderung und generische Selbstmanagementkompetenzen im Umgang mit chronischen Erkrankungen gefördert werden. Ein besonderer Fokus lag auf der Stärkung der sozialen Teilhabe in der Kommune sowie auf der Nutzung digitaler Medien zur Informationsbeschaffung. Im vorliegen- den Beitrag werden die Ergebnisse der Intervention im Bereich GK dargestellt.

\section{Methodik}

Auf der Grundlage von 11 homogenen Fokusgruppendiskussionen mit älteren Menschen und Multiplikator ${ }^{\star}$ innen wurden die Inhalte identifiziert (• Abb. 1), anschließend mit dem Health-Literacy-Konzept nach Sørensen et al. [10] verknüpft und mit adressatengerechten Gruppenübungen zu einem Bildungsprogramm einschließlich Modulhandbuch sowie zu einer Qualifikation für die Peer-Moderation zusammengesetzt (GeWinn-Interventionsentwicklung vgl. [5]). Die aus dem IROHLAProjekt abgeleiteten Handlungsempfehlungen zur Verbesserung der GK älterer Menschen wurden dabei berücksichtigt und unter Nutzung des EmpowermentAnsatzes eine Kombination aus verschiedenen Methoden verwendet [7].

Die 11 Gruppentreffen der 20-wöchigen Basisphase folgten festen Stundenbildern und stellten den Erfahrungsaustausch in den Mittelpunkt. Die etablierten Gruppen wurden nach der Basisintervention fortgeführt. In dieser 32-wöchigen Verstetigungsphase standen die Anwendung des Gelernten und die Mitwirkung in der Gemeinde im Vordergrund. Die Peers wurden in Abstimmung mit den Sozialeinrichtungen nach ihrer 

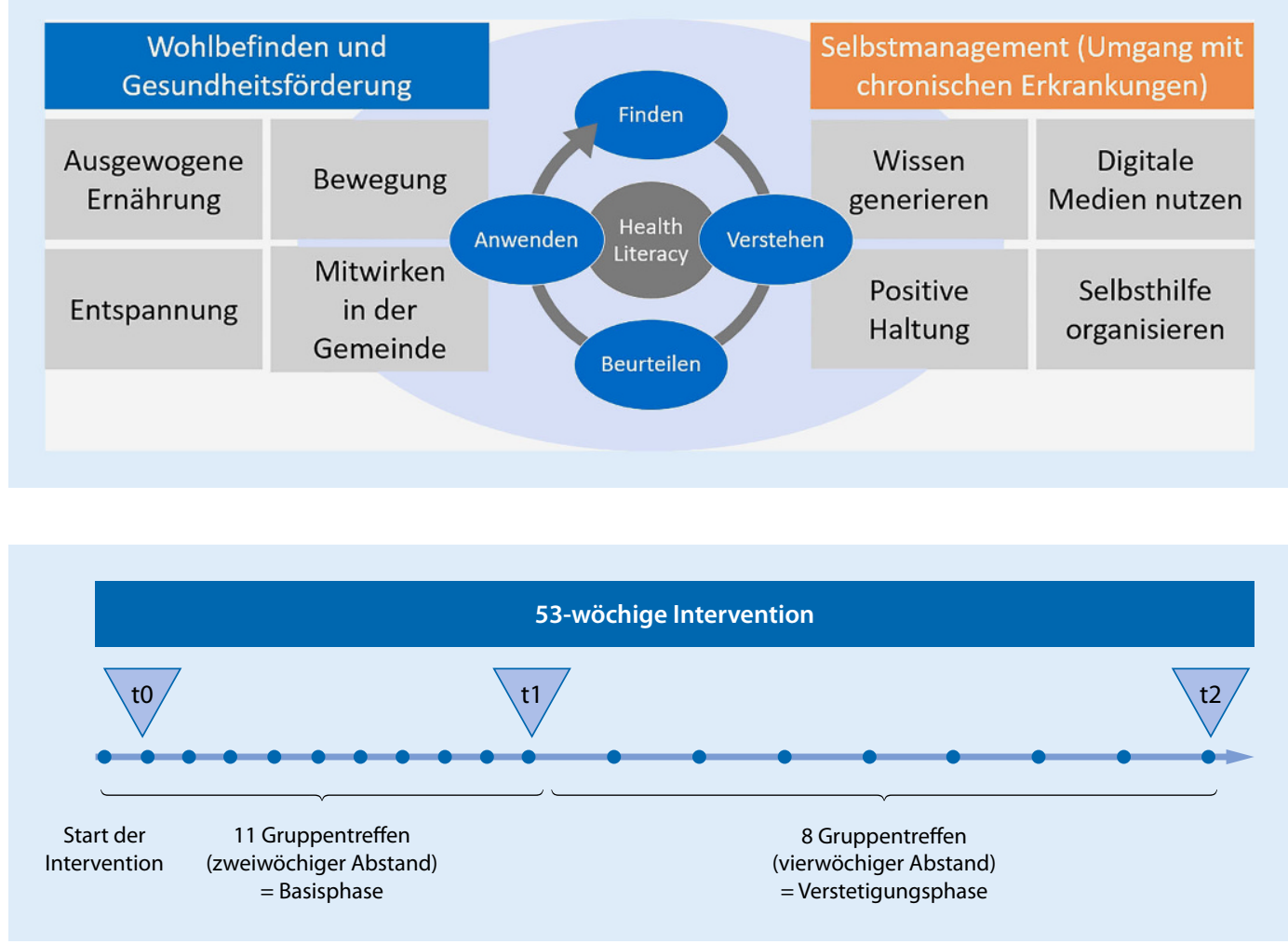

Abb. $1 \varangle$ Inhalte der Intervention

Abb. $2 \triangleleft$ Ablauf der Intervention und Datenerhebung
Gruppenerfahrung ausgewählt und in einem 2-tägigen Training bezüglich Didaktik sowie Moderation geschult. Sie fungierten nicht als Lehrende, sondern moderierten im Tandem die selbstständig arbeitenden Gruppen auf der Grundlage des Modulhandbuchs, das auf Basis der identifizierten Inhalte in den Fokusgruppendiskussionen als Schritt-für-SchrittAnleitung erstellt worden war. Außerdem wurden die Gruppenleitungen während der gesamten Intervention begleitet.

Der Interventionseffekt im Bereich GK wurde anhand eines quasiexperimentellen Evaluationsdesigns $\mathrm{zu}$ drei Messzeitpunkten (Pre-post-Vergleich) mittels der Kurzform des im Rahmen des European Health Literacy Survey entwickelten Fragebogens HLS-EU-Q16 untersucht (• Abb. 2; [4]).

Die Reliabilität (Cronbach's a für alle Indizes $>0,8)$ und Validität gelten als akzeptabel [8]. Die insgesamt 16 Items beziehen sich auf verschiedene gesundheitsrelevante Aufgaben bzw. Tätigkeiten aus den Bereichen Krankheitsbewältigung, Prävention und Gesundheitsförderung. Die Befragten schätzen dabei ein, wie einfach oder schwierig die entspre- chende Aufgabe bzw. Tätigkeit ihrer Ansicht nach ist (4 Kategorien von „sehr einfach“ bis „sehr schwierig“). Aus den Antworten wurde ein Index mit Werten von 0 (sehr geringe GK) bis 50 (sehr hohe GK) gebildet [4]. Befragten mit mehr als 3 fehlenden Werten wurde kein Indexwert zugewiesen.

Für den Index wurden Grenzwerte definiert, die 4 Levels mit unterschiedlichem GK-Niveau festlegen: Unterschieden wird zwischen (1) „inadäquater“ (0-25 Punkte), (2) „problematischer“ (26-33 Punkte), (3) „ausreichender“ (34-42 Punkte) und (4) „exzellenter“ (43-50 Punkte) GK [4].

Außerdem wurden die soziodemografischen Daten und weitere Merkmale der Teilnehmenden (TN) zum Messzeitpunkt $1\left(\mathrm{t}_{0}\right)$ mittels Fragebogen erfasst, darunter Alter, Geschlecht, Migrationshintergrund, Bildungsabschluss, Erwerbstätigkeit, Wohnumgebung, Art der Wohnung, soziales Engagement, subjektiver Gesundheitszustand sowie Anzahl und Art der chronischen Erkrankungen. Der Anteilswert für positive Verhaltensänderungen durch die Intervention wurde bei einem Signifikanzniveau von $\alpha=5 \%$ und mit einer Power von $90 \%$ $(\beta=10 \%)$ geschätzt. Aufgrund dieser Annahmen wurde der Stichprobenumfang $(n \geq 72)$ mit dem Programm $\mathrm{G}^{\star}$ Power berechnet. Die erhobenen Daten wurden mit dem Statistikprogramm IBM SPSS Statistics 26 (IBM, Armonk, NY, USA) ausgewertet. Neben einer einfaktoriellen Varianzanalyse (ANOVA) mit Messwiederholung und Bonferroni-korrigierten paarweisen Vergleichen wurden deskriptive Auswertungen und Mittelwertvergleiche zwischen den Messzeitpunkten durchgeführt, um relevante Änderungen zu prüfen. Einzelne fehlende Daten wurden akzeptiert und nicht ersetzt.

\section{Ergebnisse}

Im Verlauf einer vielschichtigen Akquise (u. a. durch Informationsveranstaltungen, Flyer, Aushänge und Presseartikel) wurden insgesamt 283 Personen (davon 250 TN und 33 Gruppenleiter*innen [GL]) rekrutiert und 25 Gruppen in den beteiligten 4 bayerischen Landkreisen sowie der Stadt Magdeburg durch die kommunalen Partner implementiert. Die 
TN wählten nach sozialen Beziehungen und Wohnortnähe ihre Gruppe selbst aus. In 8 Kommunen wurden jeweils zwei Gruppen eingerichtet.

In die Analyse der Ergebnisse (im Bereich GK) nach dem Messzeitpunkt 3 $\left(t_{2}\right)$ konnten 183 Fragebögen ( $T N=154$, $\mathrm{GL}=29$ ) eingeschlossen werden. Das durchschnittliche Alter lag bei 69,1 \pm 6,7 Jahren, wobei die GL (65,3 Jahre) im Schnitt etwas jünger als die TN (69,8 Jahre) waren. Der Männeranteil lag bei $20,8 \%(\mathrm{TN}=20,1 \%$, $\mathrm{GL}=24,1 \%)$. Hinsichtlich der Funktion als Peer-Moderator ${ }^{*}$ in bestand ein signifikanter Zusammenhang $(p<0,001)$ zum höchsten erworbenen Bildungsabschluss: $65,5 \%$ der GL hatten eine Hoch-/Fachhochschulreife bzw. einen Universitäts-/Fachhochschulabschluss erlangt, wohingegen es bei den $\mathrm{TN}$ nur etwas mehr als ein Viertel $(26,9 \%)$ waren. 68,3\% aller am Projekt Teilnehmenden ( $\mathrm{TN}=71,1 \%, \mathrm{GL}=55,2 \%)$ litten an einer oder mehr chronischen Erkrankungen $(M=1,7, S D=0,8)$, wobei Herz-Kreislauf-Erkrankungen (43\%) und Erkrankungen des Bewegungsapparats $(24 \%)$ am häufigsten genannt wurden (•Tab. 1).

Nach Abschluss des Gruppenprogramms verfügten $63,7 \%$ der TN und $72,4 \%$ der GL über eine ausreichende oder exzellente GK. Dies bedeutete eine Zunahme des Anteils der TN mit ausreichender oder exzellenter GK um 14,2\% und bei den GL um 10,5\%. Insbesondere der Anteil an TN mit exzellenter GK verdoppelte sich im Verlauf der Intervention. Der Anteil der TN mit unzureichender oder problematischer GK nahm um 17,5\% ab, der der GL um 20,0\% (• Tab. 2).

Eine ANOVA mit Messwiederholung mit Huynh-Feldt-Korrektur zeigte, dass der Messzeitpunkt einen signifikanten Einfluss auf den GK-Score aller beteiligten Senior ${ }^{*}$ innen $(F[1,91$, $346,85]=16,95, \quad p<0,001, \quad$ partielles $\left.\eta^{2}=0,085, n=183\right)$ hatte. Explizit bei den TN $(F[1,91,292,27]=14,34, p<0,001$, partielles $\left.\eta^{2}=0,086, n=154\right)$ war dies der Fall. Bonferroni-korrigierte paarweise Vergleiche verdeutlichten, dass der GK-Score der TN nach Abschluss des Gruppenprogramms ( $t_{2}: M=37,1$,

Präv Gesundheitsf 2020 · 15:399-404 https://doi.org/10.1007/s11553-020-00761-5 (c) Der/die Autor(en) 2020

F. Zastrow · A. Killenberg · C. Klünder · N. Ladebeck · S. Langner · K. Neher · M. Schimmelpfennig · T. Zeiler $\cdot$ K. Baumgarten $\cdot$ H. Hassel

\section{Intervention zur Förderung der Gesundheitskompetenz älterer Menschen - Ergebnisse des Projekts „GeWinn“}

\section{Zusammenfassung}

Hintergrund. Studien belegen, dass die Bevölkerungsgruppe der Personen ab 65 Jahren über eine unzureichende Gesundheitskompetenz (GK) verfügt. Es gibt nur wenige kommunale Angebote zur Stärkung der GK für diese Adressaten.

Ziel der Arbeit. Ziel war die Entwicklung und Erprobung einer GK-Intervention in den Bereichen Gesundheitsförderung und Selbstmanagement chronischer Erkrankungen älterer Menschen im kommunalen Setting. Material und Methoden. Es wurde ein 53-wöchiges Programm mit selbstständig arbeitenden Senior*innengruppen und PeerModeration in 14 Gemeinden Bayerns und Sachsen-Anhalts partizipativ entwickelt und umgesetzt. Die Wirkung der Intervention auf die Gesundheitskompetenz wurde anhand eines quasiexperimentellen Evaluationsdesigns zu 3 Messzeitpunkten mittels des HLS-EU-Q16 untersucht.

Ergebnisse. Von den 183 eingeschlossenen Personen (Durchschnittsalter: 69,1 $\pm 6,7$ Jahre; $20,8 \%$ männlich) verfügten $44,1 \%$ der
Teilnehmenden (TN) und 34,5\% der Gruppenleitung (GL) zu Beginn der Intervention über eine unzureichende oder problematische GK (am Ende: 36,4\% der TN, 27,6\% der GL). Eine ANOVA mit Messwiederholung ( $F$ $[1,91,346,85]=16,95, p<0,001$, partielles $\left.\eta^{2}=0,085\right)$ ergab einen statistisch signifikanten Effekt der Intervention auf die GK-Werte der Senior*innen. Ähnliche Effekte zeigten sich auch in den einzelnen Subdimensionen der GK.

Diskussion. Die Struktur der GeWinnIntervention mit dem Prinzip selbstständig arbeitender Gruppen in Kommunen eignet sich zur Steigerung der Gesundheitskompetenz älterer Menschen. Im Zuge der nachhaltigen Verankerung sollten in Zukunft verstärkt vulnerable Zielgruppen (bspw. Senior*innen mit niedrigem Sozialstatus, Migranten) angesprochen und einbezogen werden.

\section{Schlüsselwörter}

Gesundheitskompetenz - Gruppenprogramm . Kommune · Peer-Moderation - Senioren

\section{Intervention for promoting health literacy in older adults—results of the project "GeWinn"}

\section{Abstract}

Background. Health literacy $(\mathrm{HL})$ has been demonstrated to be limited among adults aged 65 and over. However, evidence-based $\mathrm{HL}$ interventions are lacking.

Objectives. The objective of this study was to develop and test a community-based HL intervention in the areas of health promotion and self-management of chronic diseases among elderly.

Materials and methods. A participatory approach was used to develop and implement a 53-week program of self-reliant, peer-moderated groups of elderly in 14 municipalities in Bavaria and Saxony-Anhalt. The intervention's effect on HL was measured by using the HLSEU-16 questionnaire three times.

Results. Of 183 elderly who completed the HL questionnaire (mean age: $69.1 \pm 6.7$ years; $20.8 \%$ male), $44.1 \%$ of the participants and $34.5 \%$ of the group leaders showed intermediate or low levels of $\mathrm{HL}$ at the start of the intervention (at the end: $36.4 \%$ of the participants, $27.6 \%$ of the group leaders). A repeated-measures analysis of variance showed statistically significant effects of time on $\mathrm{HL}$ scores $(F(1.91,346.85)=16.95$, $p<0.001, \eta^{2}=0.085$ ) over the 1 -year period. $\mathrm{HL}$ subdimensions showed similar results. Conclusions. The results indicate that the structure of the intervention is appropriate to involve elderly in self-reliant working groups to improve HL. As part of the sustained implementation of GeWinn, special attention shall be paid to reaching out to and engaging vulnerable groups (e.g. elderly with low socioeconomic status, migrants).

\section{Keywords}

Municipality · Elderly · Health literacy · Group program $\cdot$ Peer moderation 
Tab. 1 Soziodemografische Daten der Teilnehmenden (TN) und Gruppenleitung (GL), denen ein GK(Gesundheitskompetenz)-Index-Wert zugeordnet werden konnte $(n=183)$

\begin{tabular}{|c|c|c|c|c|}
\hline & Gesamt & TN $(n=154)$ & GL $(n=29)$ & $p^{a}$ \\
\hline Alter (Jahre) & $69,1 \pm 6,7$ & $69,8 \pm 6,1$ & $65,3 \pm 8,5$ & 0,035 \\
\hline \multicolumn{5}{|l|}{ Geschlecht } \\
\hline Männer & $38(20,8 \%)$ & $31(20,1 \%)$ & $7(24,1 \%)$ & 0,622 \\
\hline Migrationshintergrund & $6(3,3 \%)$ & $4(2,6 \%)$ & $2(6,9 \%)$ & 0,242 \\
\hline \multicolumn{5}{|l|}{ Bildungsabschluss } \\
\hline Haupt-/Volksschule & $77(42,1 \%)$ & $74(48,1 \%)$ & $3(10,3 \%)$ & \multirow[t]{5}{*}{$<0,001$} \\
\hline Realschule & $33(18,0 \%)$ & $27(17,5 \%)$ & $6(20,7 \%)$ & \\
\hline Fachhochschulreife & $13(7,1 \%)$ & $9(5,8 \%)$ & $4(13,8 \%)$ & \\
\hline Abitur & $12(6,6 \%)$ & $6(3,9 \%)$ & $6(20,7 \%)$ & \\
\hline Universität/Fachhochschule & $35(19,1 \%)$ & $26(16,9 \%)$ & $9(31,0 \%)$ & \\
\hline \multicolumn{5}{|l|}{ Erwerbstätigkeit } \\
\hline Ruhestand & $156(85,2 \%)$ & $135(87,7 \%)$ & $21(72,4 \%)$ & 0,039 \\
\hline \multicolumn{5}{|l|}{ Wohnumgebung } \\
\hline Städtisch & $72(39,3 \%)$ & $63(40,9 \%)$ & $9(31,0 \%)$ & \multirow[t]{2}{*}{0,304} \\
\hline Ländlich & $105(57,4 \%)$ & $85(55,2 \%)$ & $20(69,0 \%)$ & \\
\hline \multicolumn{5}{|l|}{ Art der Wohnung } \\
\hline Eigenheim & $141(77,0 \%)$ & $116(75,3 \%)$ & $25(86,2 \%)$ & \multirow[t]{3}{*}{0,677} \\
\hline Mietwohnung & $37(20,2 \%)$ & $33(21,4 \%)$ & $4(13,8 \%)$ & \\
\hline Pflegeheim & $2(1,1 \%)$ & $2(1,3 \%)$ & 0 & \\
\hline Sozial engagiert & $88(48,1 \%)$ & $64(41,6 \%)$ & $24(82,8 \%)$ & $<0,001$ \\
\hline \multicolumn{5}{|l|}{ Subjektiver Gesundheitszustand } \\
\hline Ausgezeichnet & $5(2,7 \%)$ & $3(1,9 \%)$ & $2(6,9 \%)$ & \multirow[t]{5}{*}{0,005} \\
\hline Sehr gut & $30(16,4 \%)$ & $20(13,0 \%)$ & $10(34,5 \%)$ & \\
\hline Gut & $122(66,7 \%)$ & $106(68,8 \%)$ & $16(55,2 \%)$ & \\
\hline Weniger gut & $21(11,5 \%)$ & $21(13,6 \%)$ & 0 & \\
\hline Schlecht & $2(1,1 \%)$ & $2(1,3 \%)$ & 0 & \\
\hline $\begin{array}{l}\text { Mindestens eine chronische } \\
\text { Erkrankung }\end{array}$ & $125(68,3 \%)$ & $109(71,1 \%)$ & $16(55,2 \%)$ & \multirow[t]{6}{*}{0,084} \\
\hline Keine & $56(30,6 \%)$ & $43(27,9 \%)$ & $13(44,8 \%)$ & \\
\hline Eine & $61(33,3 \%)$ & $52(33,8 \%)$ & $9(31,0 \%)$ & \\
\hline Zwei & $37(20,2 \%)$ & $31(20,1 \%)$ & $6(20,7 \%)$ & \\
\hline Drei & $25(13,7 \%)$ & $24(15,6 \%)$ & $1(3,4 \%)$ & \\
\hline Vier oder mehr & $2(1,1 \%)$ & $2(1,3 \%)$ & 0 & \\
\hline
\end{tabular}

$S D=6,9)$ signifikant höher lag als zu Beginn ( $\left.\mathrm{t}_{0}: \quad M=34,3, \quad S D=7,8\right)$ und nach Abschluss der Basisintervention $\left(t_{1}: M=35,2, S D=7,4\right)$. Die Effektstärken $f$ nach Cohen (1988) entsprachen jeweils einem mittleren Effekt ( $\left.f_{\text {Studienteilnehmende }}=0,305, f_{\mathrm{TN}}=0,307\right) . \mathrm{Zu}$ erwähnen ist, dass die Zunahme der GK nur bei der Gruppe der Frauen signifikant $(F \quad[1,88,270,96]=15,86$, $p<0,001$, partielles $\eta^{2}=0,099, n=145$ ) bei einem mittleren bis starken Effekt $\left(f_{\text {Frauen }}=0,331\right)$ war, bei den Männern
$M=36,9, S D=8,6)$ signifikant höher als zu Beginn.

Bezüglich der 4 Stadien der Informationsverarbeitung Gesundheitsinformationen finden $\left(\mathrm{t}_{0}: M=33,5, S D=9,9\right.$; $\left.\mathrm{t}_{2}: M=36,0, S D=8,8\right)$, verstehen $\left(\mathrm{t}_{0}\right.$ : $\left.M=38,5, S D=7,6 ; \mathrm{t}_{2}: M=40,7, S D=6,8\right)$, beurteilen $\left(\mathrm{t}_{0}: \quad M=31,0, \quad S D=9,1 ; \mathrm{t}_{2}\right.$ : $M=34,3, S D=8,6)$ und anwenden $\left(\mathrm{t}_{0}\right.$ : $M=33,2, S D=9,2 ; \mathrm{t}_{2}: M=36,2, S D=8,3$ ) bewirkte die "GeWinn“-Intervention eine signifikante Verbesserung der GKWerte aller Beteiligten auf allen Stadien, besonders bei den Stadien Beurteilen und Anwenden (•Tab. 2 und 3).

\section{Diskussion}

Mit der GeWinn-Intervention kann die GK von Menschen ab 60 Jahren wirksam gefördert werden. Im Durchschnitt erreichten alle Beteiligten vor der Intervention einen GK-Wert von 34,7 $(S D=7,8)$ und nach der Intervention einen GKWert von 37,4 $(S D=6,9)$. Diese Werte liegen über dem GK-Durchschnitt der deutschen Bevölkerung über 65 Jahren $(M=32,8, S D=6,2$; [9]). Die Studienteilnehmenden verfügten also bereits zu Beginn über ein besseres GK-Niveau als im deutschen Mittel. Dies könnte darin begründet liegen, dass programmaffine Senior*innen eher sozial engagiert und besser gebildet sind und sich gesünder verhalten. Dem widerspricht jedoch die hohe Zahl der chronisch Erkrankten $(68,3 \%)$, die deutlich über dem deutschen Durchschnitt der über 65-Jährigen (Frauen: 60,2\%, Männer: 53,8\%) lag und als weiterer Risikofaktor für ein schlechtes GK-Niveau gilt [6]. Allerdings schätzen die meisten trotz chronischer Erkrankung ihren Gesundheitszustand als recht gut ein: Nur 12,6\% der Senior*innen der GeWinn-Intervention gaben einen weniger guten oder schlechten Gesundheitszustand an (-Tab. 1).

Die Wirkung der Intervention im Bereich GK konnte nur bei den teilnehmenden Frauen signifikant nachgewiesen werden, bei den Männern hingegen nicht. Der Stichprobenumfang bestand jedoch nur aus etwa einem Fünftel (38 von 183) an Männern. Bei einem größeren Anteil an Männern im Stichprobenumfang könnte es wahrscheinlicher sein, 
Tab. 2 Gesundheitskompetenzniveaus in 4 Levels zu allen 3 Messzeitpunkten in Prozent $(n=183)$

\begin{tabular}{|c|c|c|c|c|c|c|c|c|c|}
\hline & & \multicolumn{4}{|c|}{ Teilnehmer $(n=154)$} & \multicolumn{4}{|c|}{ Gruppenleiter $(n=29)$} \\
\hline & & 1 & 2 & 3 & 4 & 1 & 2 & 3 & 4 \\
\hline \multirow[t]{3}{*}{ GK-Niveau } & to & 8,4 & 35,7 & 43,5 & 12,3 & 6,9 & 27,6 & 44,8 & 20,7 \\
\hline & $t 1$ & 9,1 & 34,4 & 44,2 & 12,3 & 3,4 & 34,5 & 48,3 & 13,8 \\
\hline & t2 & 3,9 & 32,5 & 39,0 & 24,7 & 0 & 27,6 & 37,9 & 34,5 \\
\hline \multicolumn{10}{|l|}{ Bereich } \\
\hline \multirow{3}{*}{$\begin{array}{l}\text { Krankheits- } \\
\text { bewältigung }\end{array}$} & to & 11,0 & 32,5 & 29,2 & 22,7 & 6,9 & 34,5 & 24,1 & 34,5 \\
\hline & $t 1$ & 9,1 & 37,0 & 24,7 & 27,3 & 6,9 & 37,9 & 17,2 & 37,9 \\
\hline & $t 2$ & 5,8 & 29,9 & 30,5 & 31,8 & 0 & 31,0 & 20,7 & 48,3 \\
\hline \multirow[t]{3}{*}{ Prävention } & to & 12,3 & 33,8 & 36,4 & 16,2 & 10,3 & 27,6 & 37,9 & 24,1 \\
\hline & $t 1$ & 8,4 & 37,0 & 37,7 & 13,6 & 10,3 & 31,0 & 37,9 & 17,2 \\
\hline & $t 2$ & 3,9 & 37,7 & 29,9 & 26,6 & 13,8 & 24,1 & 27,6 & 34,5 \\
\hline \multirow{3}{*}{$\begin{array}{l}\text { Gesundheits- } \\
\text { förderung }\end{array}$} & to & 14,9 & 38,3 & 26,0 & 17,5 & 6,9 & 37,9 & 24,1 & 27,6 \\
\hline & $t 1$ & 18,8 & 39,6 & 22,1 & 18,2 & 13,8 & 37,9 & 17,2 & 31,0 \\
\hline & $t 2$ & 11,7 & 43,5 & 16,9 & 25,3 & 10,3 & 27,6 & 27,6 & 34,5 \\
\hline \multicolumn{10}{|l|}{ Stufe } \\
\hline \multirow[t]{3}{*}{ Finden } & to & 22,7 & 33,1 & 27,9 & 11,7 & 13,8 & 34,5 & 17,2 & 31,0 \\
\hline & $t 1$ & 22,7 & 36,4 & 24,0 & 13,6 & 3,4 & 44,8 & 24,1 & 24,1 \\
\hline & $t 2$ & 14,3 & 36,4 & 31,8 & 16,2 & 10,3 & 31,0 & 31,0 & 27,6 \\
\hline \multirow[t]{3}{*}{ Verstehen } & to & 3,9 & 24,7 & 40,9 & 27,9 & 6,9 & 24,1 & 41,4 & 27,6 \\
\hline & $t 1$ & 5,8 & 24,7 & 36,4 & 32,5 & 3,4 & 27,6 & 44,8 & 24,1 \\
\hline & $t 2$ & 2,6 & 18,2 & 34,4 & 42,2 & 0 & 31,0 & 27,6 & 41,4 \\
\hline \multirow[t]{3}{*}{ Beurteilen } & to & 28,6 & 33,8 & 27,3 & 9,1 & 10,3 & 41,4 & 27,6 & 20,7 \\
\hline & $t 1$ & 18,8 & 49,4 & 16,2 & 13,6 & 13,8 & 44,8 & 17,2 & 24,1 \\
\hline & $t 2$ & 18,2 & 45,5 & 15,6 & 18,2 & 3,4 & 34,5 & 27,6 & 34,5 \\
\hline \multirow[t]{3}{*}{ Anwenden } & to & 16,9 & 49,4 & 17,5 & 15,6 & 13,8 & 55,2 & 10,3 & 20,7 \\
\hline & $t 1$ & 11,7 & 49,4 & 20,1 & 18,2 & 6,9 & 58,6 & 17,2 & 17,2 \\
\hline & $t 2$ & 8,4 & 48,1 & 16,9 & 26,0 & 3,4 & 55,2 & 6,9 & 34,5 \\
\hline
\end{tabular}

dass der Effekt signifikant wird. Männer gehören neben Alleinlebenden zu einem schwer erreichbaren Personenkreis. Deswegen ist es wichtig, diese Gruppen bei der Akquise in zukünftigen Interventionen noch stärker anzusprechen.

Die selbstständig arbeitenden Senior*innengruppen durchliefen das methodisch vielfältige Gruppenprogramm eigenständig und wiederholten die $\mathrm{Ge}$ nerierung gesundheitsbezogener Informationen im Lauf der Intervention mehrmals. Sie waren hinsichtlich Bildung und GK sehr heterogen. Der Peerto-Peer-Ansatz scheint in der Förderung der GK und sicher auch im Zusammenhalt der Gruppen eine zentrale Rolle einzunehmen [2, 7]. Eine Auswertung der Akzeptanzerhebung (hier nicht dar- gestellt, Publikation in Vorbereitung) zeigt, dass das Gruppenprogramm auf 87,9\% der Beteiligten würden das Programm weiterempfehlen und $75,7 \%$ haben das Gefühl, durch die GeWinnTreffen aktiv am Leben teilgenommen zu haben.

Die Möglichkeit, als Gruppenleitung aktiv zu sein, motivierte gerade die Studienteilnehmenden mit höherem Bildungsabschluss. Sie hatten zudem eine höhere GK und weniger chronische Erkrankungen. Die GL fungierten bei komplexen Themen bei den TN mit wenig bzw. keiner Vorbildung als Türöffner. Auf diesem Weg erschlossen sich für diese TN neue Informationsund Kommunikationsmöglichkeiten. So eine gute bis sehr gute Resonanz trifft: können die teils großen Unterschiede im GK-Niveau der TN und GL als Stärke des Gruppenprogramms angesehen werden, da hierüber innerhalb der Gruppen ein Austausch zwischen Menschen mit deutlichen Bildungsunterschieden und ganz individuellen Stärken stattfand. Hinzu kommt, dass auch die GL vom Gruppenprogramm profitierten.

\section{Schlussfolgerung}

Der Ansatz und die Struktur der vorgelegten GeWinn-Intervention mit selbstständig arbeitenden Gruppen und PeerModeration scheinen zu einem wirksamen Konzept beizutragen, die GK in der Gruppe älterer Menschen mit teils chronischen Erkrankungen zu verbessern. Die hohe Akzeptanz unter den Senior*innen sichert zudem eine Beteiligung der Zielgruppe an kommunalen Angeboten und somit ein Entgegenwirken sozialer Isolation zu.

$\mathrm{Zu}$ überprüfen bleibt, ob durch eine Fortführung der Intervention die GK der Senior*innen weiter verbessert werden kann und dies ferner auf eine gute Resonanz bei den TN stößt. Eine selbstständige Fortführung der Gruppen nach der Pilotstudie ist mit den Gruppen und Praxispartnern (Landkreisen und Senioreneinrichtungen) vereinbart.

\section{Fazit für die Praxis}

- Mit der GeWinn-Intervention kann die Health Literacy älterer Menschen (über 60 Jahren) wirksam gefördert werden.

- Nach Abschluss des Gruppenprogramms verfügten $63,7 \%$ der Teilnehmenden (TN) und 72,4\% der Gruppenleiter*innen (GL) über eine ausreichende oder exzellente Gesundheitskompetenz (GK). Dies bedeutet eine Zunahme des Anteils mit ausreichender oder exzellenter GK bei den TN um 14,2\% und bei den GL um 10,5\%. Insbesondere der Anteil an TN mit exzellenter GK hat sich im Verlauf der Intervention verdoppelt.

- Bei zukünftigen Interventionen sollten schwer erreichbare Personengruppen, wie z. B. Männer oder 


\section{Originalarbeit}

Tab. 3 F-Statistik der Varianzanalyse der Gesundheitskompetenz (GK) der 3 Bereiche und 4 Stadien $(n=183)$

\begin{tabular}{|c|c|c|c|c|c|c|c|c|c|}
\hline & \multicolumn{3}{|c|}{ Teilnehmer $(n=154)$} & \multicolumn{3}{|c|}{ Gruppenleiter ( $n=29$ ) } & \multicolumn{3}{|c|}{ Alle $(n=183)$} \\
\hline & F-Wert ${ }^{a}$ & $P^{b}$ & $\eta^{2 c}$ & F-Wert ${ }^{\mathrm{a}}$ & $P^{b}$ & $n^{2 c}$ & F-Wert ${ }^{a}$ & $P^{b}$ & $n^{2 c}$ \\
\hline GK-Score & 14,34 & $<0,001$ & 0,086 & 3,03 & 0,057 & 0,098 & 16,95 & $<0,001$ & 0,085 \\
\hline \multicolumn{10}{|l|}{ Bereich } \\
\hline Krankheitsbewältigung & 9,88 & $<0,001$ & 0,065 & 3,81 & 0,028 & 0,120 & 12,88 & $<0,001$ & 0,070 \\
\hline Prävention & 12,16 & $<0,001$ & 0,078 & 1,44 & 0,245 & 0,051 & 12,21 & $<0,001$ & 0,067 \\
\hline Gesundheitsförderung & 7,21 & 0,001 & 0,048 & 1,95 & 0,152 & 0,067 & 8,88 & $<0,001$ & 0,050 \\
\hline \multicolumn{10}{|l|}{ Stadium } \\
\hline Finden & 7,77 & 0,001 & 0,053 & 0,22 & 0,804 & 0,008 & 7,68 & 0,001 & 0,044 \\
\hline Verstehen & 9,76 & $<0,001$ & 0,063 & 2,11 & 0,130 & 0,070 & 11,39 & $<0,001$ & 0,061 \\
\hline Beurteilen & 12,61 & $<0,001$ & 0,081 & 2,95 & 0,061 & 0,095 & 15,10 & $<0,001$ & 0,080 \\
\hline Anwenden & 10,49 & $<0,001$ & 0,065 & 3.59 & 0,034 & 0,114 & 13,13 & $<0,001$ & 0,068 \\
\hline
\end{tabular}

${ }^{\mathrm{a}} \mathrm{F}$-Wert gibt Varianzunterschiede bei zwei unabhängigen Stichproben an

${ }^{\mathrm{b}}$ Zweiseitige Signifikanz $p<0,05$

${ }^{c}$ Partielles $\eta^{2}$ gibt den Anteil der aufgeklärten Varianz auf der Stichprobenebene an

\section{Alleinlebende, bei der Akquise stär- ker angesprochen werden.}

\section{Korrespondenzadresse}

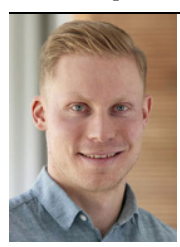

Felix Zastrow

Institut für angewandte

Gesundheitswissenschaften

(laG), Hochschule für

angewandte Wissenschaften

Coburg

Friedrich-Streib-Str. 2 ,

96450 Coburg, Deutschland

felix.zastrow@hs-coburg.de

Funding. Open Access funding provided by Projekt DEAL.

\section{Einhaltung ethischer Richtlinien}

Interessenkonflikt. F. Zastrow, A. Killenberg, C. Klünder, N. Ladebeck, S. Langner, K. Neher, M. Schimmelpfennig, T.Zeiler, K. Baumgarten und H. Hassel geben an, dass kein Interessenkonflikt besteht.

Die Ethikkommission der Hochschule für angewandte Wissenschaften Coburg hat das Projektvorhaben nach den ethischen Richtlinien geprüft und für ethisch unbedenklich erklärt. Von allen beteiligten Teilnehmenden liegt eine Einverständniserklärung vor. Für die aufgeführten Studien gelten die jeweils dort angegebenen ethischen Richtlinien.

Open Access. Dieser Artikel wird unter der Creative Commons Namensnennung 4.0 International Lizenz veröffentlicht, welche die Nutzung, Vervielfältigung Bearbeitung, Verbreitung und Wiedergabe in jeglichem Medium und Format erlaubt, sofern Sie den/die ursprünglichen Autor(en) und die Quelle ordnungsgemäß nennen, einen Link zur Creative Commons Lizenz beifügen und angeben, ob Änderungen vorgenommen wurden.
Die in diesem Artikel enthaltenen Bilder und sonstiges Drittmaterial unterliegen ebenfalls der genannten Creative Commons Lizenz, sofern sich aus der Abbildungslegende nichts anderes ergibt. Sofern das betreffende Material nicht unter der genannten Creative Commons Lizenz steht und die betreffende Handlung nicht nach gesetzlichen Vorschriften erlaubt ist, ist für die oben aufgeführten Weiterverwendungen des $\mathrm{Ma}$ terials die Einwilligung des jeweiligen Rechteinhabers einzuholen.

Weitere Details zur Lizenz entnehmen Sie bitte der Lizenzinformation auf http://creativecommons.org/ licenses/by/4.0/deed.de.

\section{Literatur}

1. Berkman ND, Sheridan SL, Donahue KE, Halpern DJ, Crotty K (2011) Low health literacy and health outcomes: an updated systematic review. Ann Intern Med 155(2):97-107

2. Haslbeck J (2015) Das Stanford Selbstmanagementprogramm „Gesund und aktiv leben" (Evivo) J Gesundhförd 3:22-23

3. Haslbeck J (2017) Medication Literacy - Gesundheitskompetenz, chronischer Krankheit und Selbstmanagement bei Medikamenten. In: Schaeffer D, Pelikan JM (Hrsg) Health Literacy: Forschungsstand und Perspektiven. Hogrefe, Bern, S259-275

4. HLS-EU Consortium (2012) Comparative report of health literacy in eight EU member states. The European health literacy survey HLS-EU (second revised and extended version, date july 22th, 2014). http://www.healthliteracyeurope. net. Zugegriffen:23. Januar 2020

5. Killenberg A, Ladebeck N, Baumgarten K, Hassel H (2017) Gesundheitskompetenz im Alter fördern Partizipative Interventionsentwicklung im Projekt "GeWinn". Public Health Forum 25(1):90-93

6. Lange C, Lampert T (2011) Daten und Fakten: Ergebnisse der Studie "Gesundheit in Deutschland aktuell 2009". https://www. rki.de/DE/Content/Gesundheitsmonitoring/ Gesundheitsberichterstattung/GBEDownloadsB/ GEDA09.pdf.Zugegriffen: 18. Juni 2019

7. Rohde T, Kolpatzik K, Winter AF (2015) Förderung der Gesundheitskompetenz älterer Menschen. Ansatzpunkte auf Basis des EU-Projektes IROHLA
Bundesgesundheitsblatt Gesundheitsforschung Gesundheitsschutz 58:958-965

8. Schaeffer D, Vogt D, Berens EM et al (2016) Gesundheitskompetenz der Bevölkerung in Deutschland. Universität Bielefeld, Bielefeld

9. Schaeffer D, Vogt D, Berens EM et al (2016) Health Literacy in Deutschland. In: Schaeffer D, Pelikan JM (Hrsg) Health Literacy: Forschungsstand und Perspektiven. Hogrefe, Göttingen, S129-143

10. Sørensen K, Broucke SVD, Fullam J et al (2012) Health literacy and public health: a systematic review and integration of definitions and models. BMC Public Health 12:80

11. Tokuda Y, Doba N, Butler JP et al (2009) Health literacy and physical and psychological wellbeing in Japanese adults. Patient Educ Couns 75(3):411-417

12. Zok K (2014) Unterschiede bei der Gesundheitskompetenz. Ergebnisse einer bundesweiten Repräsentativ-Umfrage unter gesetzlich Versicherten. WIdO Monit 11:1-12 